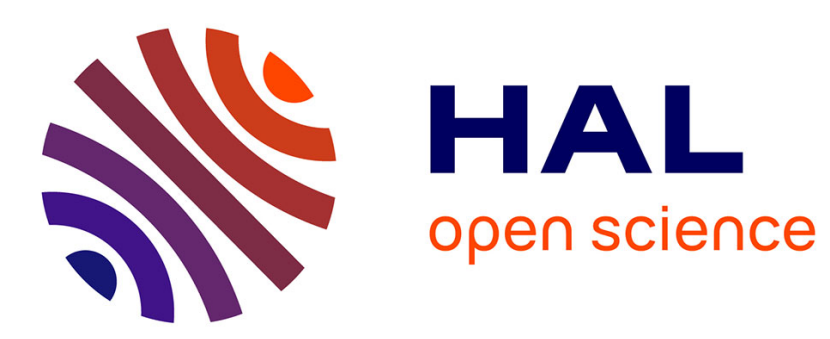

\title{
A few myths about Telco and OTT models
}

\author{
Emmanuel Bertin, Noel Crespi, Michel L'Hostis
}

\section{To cite this version:}

Emmanuel Bertin, Noel Crespi, Michel L'Hostis. A few myths about Telco and OTT models. ICIN 2011: 15th International Conference on Intelligence in Next Generation Networks: From Bits to Data, from Pipes to Clouds, Oct 2011, Berlin, Germany. pp.6 - 10, 10.1109/ICIN.2011.6081106 . hal-01303630

\section{HAL Id: hal-01303630 \\ https://hal.science/hal-01303630}

Submitted on 18 Apr 2016

HAL is a multi-disciplinary open access archive for the deposit and dissemination of scientific research documents, whether they are published or not. The documents may come from teaching and research institutions in France or abroad, or from public or private research centers.
L'archive ouverte pluridisciplinaire HAL, est destinée au dépôt et à la diffusion de documents scientifiques de niveau recherche, publiés ou non, émanant des établissements d'enseignement et de recherche français ou étrangers, des laboratoires publics ou privés. 


\title{
A few myths about Telco and OTT models
}

\author{
Emmanuel Bertin ${ }^{1,2}$ \\ emmanuel.bertin@orange- \\ ftgroup.com
}

\author{
Noel Crespi ${ }^{2}$ \\ noel.crespi@it-sudparis.eu
}

\author{
Michel L'Hostis ${ }^{3}$ \\ michel.1hostis@orange-ftgroup.com
}

\author{
1 - Orange Labs - 42, rue des Coutures 14066 Caen, France \\ 2 - Institut Telecom, Telecom SudParis, CNRS 5157 - 9, rue Charles Fourier 91011 Evry, France \\ 3 - Orange Labs - 2, avenue Pierre Marzin 22307 Lannion, France
}

\begin{abstract}
Over the next few years, we will witness radical changes in the way users consume communication services. Two of the most visible trends are the Over-The-Top (OTT) models, pushed by webcos, i.e. the major companies driving the offering of web services, and the telco model, favored by telecommunication network operators. Are they that different? With an analysis of the differences and the commonalities between these two models, this paper unveils the reality about some of the myths surrounding OTT and the telcos' models.
\end{abstract}

\section{INTRODUCTION}

Telco and OTT models are sometime seen as fundamentally different actors, whose only common point would be to (quite accidentally) compete economically, since the latter have disrupted the market of the former. In this paper, we intend to debunk a few myths about this competition between telcos and OTT models, thereby continuing the ICIN tradition [1]. We define here an OTT provider as a service provider that offers telecom services, but that neither operates a telecom network nor leases networking capabilities from a telecom operator, relying only on the worldwide Internet network. In the rest of the paper, we will successively review some of the most common assertions about telcos and OTT providers, and conclude with some future scenarios.

\section{MYTH \#1: THEIR ARCHITECTURES ARE INTRINSICALLY DIFFERENT}

Concerning communication services, OTT and telco architectures are often considered to be intrinsically different. Telco communication architectures are usually divided into three independent layers:

- A network layer, with access and core networks;

- A control layer, with AAA and service triggering, as well as communications control (the key historical telco service); and

- An application layer, with service platforms that provide supplementary value-added services on top of the control layer, using protocols such as SIP (an ISC interface).

The control layer is here tightly linked to the network layer. First, the control layer might pilot the network layer. For instance, in the IMS architecture, the treatment of the media flows (e.g. QoS) depends on the signaling layer. Second, information from the network layer might be reused by the control layer. For instance, the network authentication is sometimes used by the control layer to personalize services. When launching the operator web portal, the customer of a network access offering is thereby directly recognized, without having to authenticate him/herself (implicit authentication).

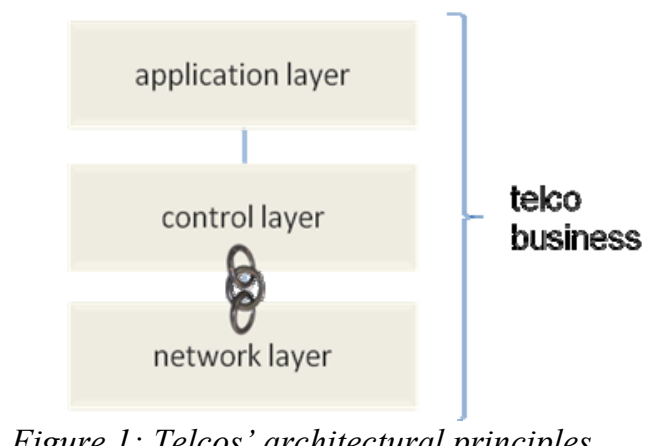

Interestingly OTT models have layers that are quite similar:

- A control layer, with identity management, access control and API exposure, as well as the key OTT services (e.g. search for Google, communication control for Skype or social networking for Facebook);

- An application layer, where third-parties provide valueadded services on top of the control layer, using APIs; and

- A network layer, similar to that of the telcos. 


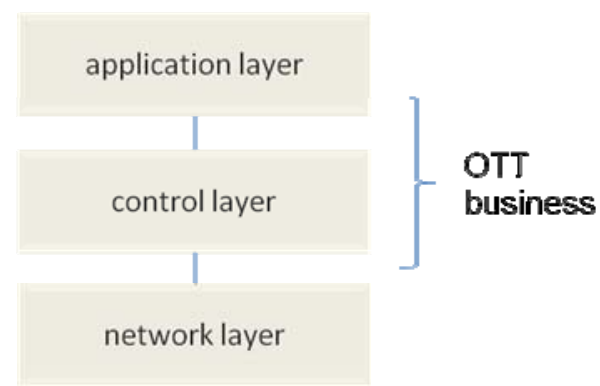

Figure 2: OTT architectural principles

The control layer here is fully independent from the network layer. This implies two main drawbacks. First, an independent control layer cannot decide the network policies to apply to IP flows. Second, it cannot make use of networklevel information.

These drawbacks have, however, been circumvented by OTT businesses. Today, the network bandwidth is no longer an issue in terms of offering real-time services over wirelineaccess networks, and this will also probably be the case over $4 \mathrm{G}$ wireless networks, except for overloaded cells. Moreover, their attraction powers as well as the regulatory bodies prevent the operators that operate the network layer from applying policies that will deteriorate the access to their services. Finally, the OTT managed to function without information arriving from the network, relying only on the endpoints. For example, with implicit authentication, the same user experience can be rendered using web browser cookies.

In conclusion, their architectures are functionally close, the major difference being the link between the network and the control layers. This architectural difference implies a business one; in terms of sales, telcos traditionally offer their services to their local customers (i.e. the clients of their access networks), whereas the natural market of OTTs is globally open and not limited to a specific customer base. In that sense, OTT players have started to de-perimeterise the service from the access [2].

\section{MYTH \#2: TELCOS ARE USED TO OPERATING IN A CLOSED WORLD WHILE OTTS PROMOTE AN OPEN ONE}

Telcos certainly are supporters of a walled-garden paradigm, with more or less success (for example, think of WAP, the French Minitel, or the Japanese i-mode). But are OTTs truly promoting an open world?

Interworking is in the telco's DNA. Their operators are used to operating a wide range of devices, platforms and equipment from different manufacturers, and to ensure that they work together to provide the required telecom services, relying on standardized protocols between all these elements. For their traditional voice service, every operator interoperates with all the others, enabled by a strong standardization and profiling emphasis, and achieved through peering agreements. This interworking based on open standards has proven to be a very efficient way to interconnect tens of thousands of network operators throughout the world, complete with transparent revenue sharing [3]. The application layer is the only one that is minimally interoperable between operators.

The difficult interoperability of the application layer could be analyzed as a consequence of the network effect. As operators address only the customer of a given territory, peering is a solution that could extend their network by extending their geographical coverage. However, it should be noted that such interworking effort has not yet been widely adopted by VoIP services. Interworking between the different VoIP operators is still mainly done through the PSTN, partially for regulatory reasons. Standardization is achieved, but profiling recommendations and peering agreements are not.

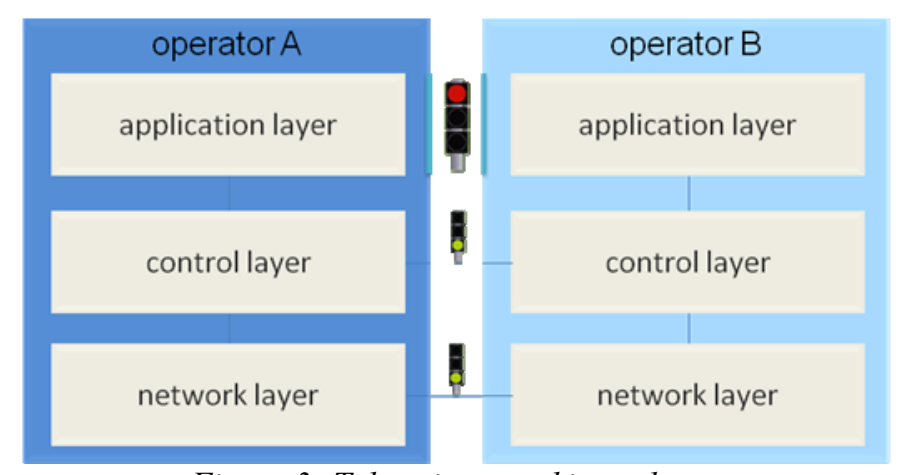

Figure 3: Telcos interworking scheme

Contrarily, OTT models cannot interoperate at all, neither at the control level, nor at the application level. A phone call from a T-Mobile user to an Orange user is fully natural, but adding a MySpace user to my Facebook friends is anything but natural and easy. Since OTT providers address a global market and their customers are more volatile than operators', their interest in peering is simply lower.

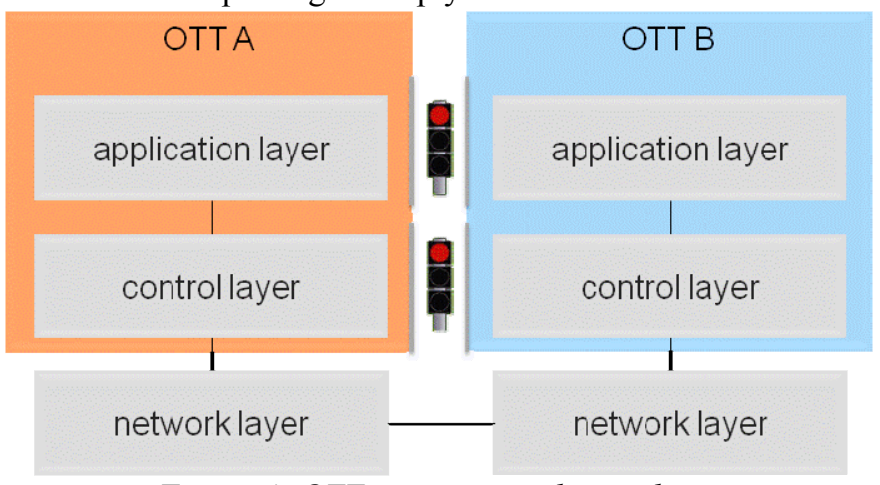

Figure 4: OTT non-interworking scheme

Moreover, application developers are locked to the control layer of their OTT provider. An iPhone app will never run on Android, even where operators attempted with Parlay or JAIN to provide common APIs to their control layers. Some OTTs have even fully adopted the walled garden paradigm. Apple, for instance, maintains a total control on the applications built over its control layer, requiring approval for each app before publication and even retaining (and using!) the capability to remove them after purchase (not a popular practice). 
In sum, interoperability is a key telco strength, even more so than openness. Interoperability is achieved between peers, through standards and agreements; it requires detailed specification and tight control. Openness means that smaller actors can use your systems in a way that you did not determine beforehand; it is giving away some power in order gain more. Concerning OTTs, their level of openness depends on their power (competitive advantages) and on their strategies. For example, Apple's customers support the walled-garden paradigm because they appreciate the competitive advantage of their very usable devices and interfaces. Openness is not a key factor to gain or to retain customers, but a strategic option.

\section{MYTH \#3: OTTS HAVE NO BOUNDARIES}

Recently, the telco industry forged the telco 2.0 paradigm to compete with OTT technologies by addressing global markets and not only local ones (i.e. with service offers linked to network access). This was possible by basically making an opening for APIs on top of the telco control layer to attract developers that will build a full application ecosystem, in the image of the OTT walled garden. It is an effort towards an open architecture, but open architecture is not enough to succeed on the market, as mentioned above. The telco 2.0 strategy may address the needs of certain business markets, and it is also very valuable to inside operators as a driver towards service-oriented architectures. This paradigm encourages telcos to modularize their service platforms, and to offer APIs that can be reused internally by other services from the same operator. However, it barely de-perimeterises services and till now has had little success against OTT offerings.

Telcos are indeed limited to a given geographical territory, due to their adherence to an access network, as seen in the first section. Meanwhile, major OTTs have extended their strategy towards devices.

In the telco world, terminals are considered as network and call control endpoints; their conception is generally outside the operators' core business. At the same time, one of the key strengths of OTT models is their control of the device control layer, e.g. through Android or iOS. This control enables them to blur the border between local and remote, by enabling the user to seamlessly synchronize local and remote data (e.g. with Apple iCloud or Google Chrome OS).

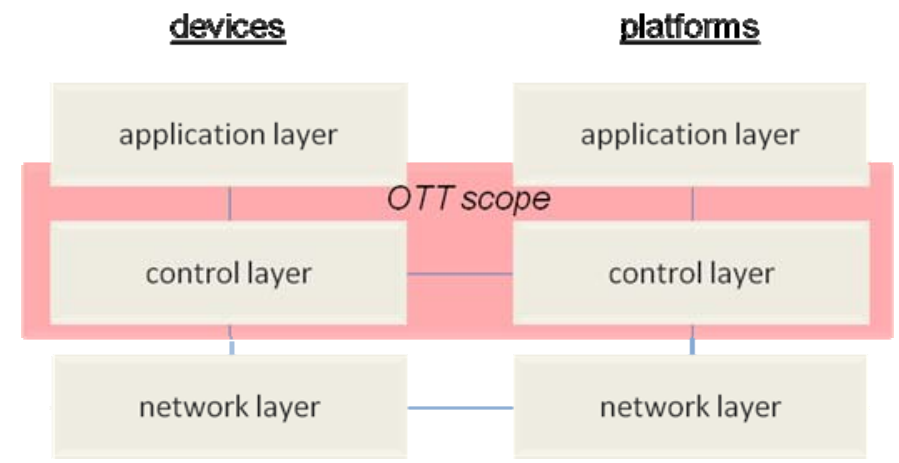

Figure 5: OTTs operational scope
In conclusion, the telco historical boundary between devices and platforms (User-Network Interface) is certainly fading, but a new one is rising up. The resulting OTT ecosystems remain independent and non-interoperable. Apple iCloud is, for instance only working with Apple devices. On the internet, customers have accepted moving very quickly to a single solution ("winner takes all" paradigm), as the migration cost is relatively low (e.g. changing some habits, reentering data). Customers will probably be much less amenable to only buying products from a given ecosystem business or mass market. Telcos have here the potential to bring standardization and interoperability.

\section{MYTH \#4: NET-NEUTRALITY IS THE PANACEA}

Net-neutrality aims to prevent operators from using their network assets to distinguish between service actors. Figure 6 represents this principle, with a Swiss flag figuring neutrality.

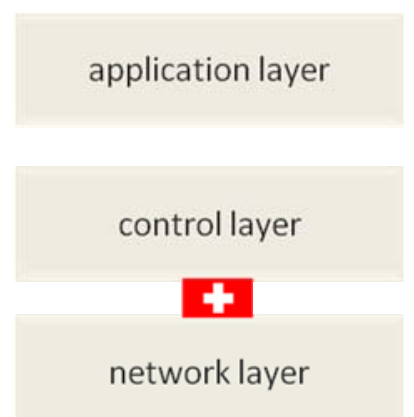

Figure 6: Net neutrality principle

But, as we have discussed above, a symmetrical regulation should extend this principle to the control layer: Webcos should not be able to make use of their control layer to distinguish between application providers. Moreover, the OTT control layer should be interoperable, at least to a certain extent. This certainly will imply some standardization requirements. Figure 7 symbolizes this need for interoperability with plugs and sockets.

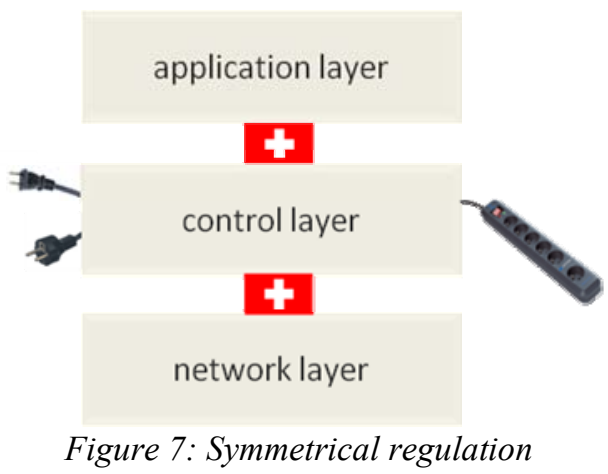

The French regulator body has, for instance, decided to study the current difficulties encountered by users who wish to migrate their digital environment (personal data, applications, etc.) when changing their mobile device [4]. Tim $\mathrm{Wu}$, who 
forged the concept of net-neutrality and is currently senior advisor to the FCC, is now advocating a separation principle: "those who develop information, those who control the network infrastructure on which it travels, and those who control the tools or venues of access must be kept apart from one another." This would prevent, for instance, an application vendor from discriminating between the applications it publishes, or a search engine from disfavouring competing sites.

\section{MYTH \#5: TELEPHONY IS FOREVER}

The telephony use case has changed very little since Bell's time. On the PSTN, an ISDN, a PLMN or via an IP Multimedia Subsystem, Alice still calls Bob by composing Bob's phone number on her device, Bob's device rings, Bob takes the call and both talk together. Alice is usually charged for that. This basic use case is enriched by an extensive range of features called Supplementary Services, which include call forwarding, call barring, call hold, call waiting, calling line identification/presentation/restriction, etc. A key telco asset here is the numbering: the capability to link a public phone number to a device and to route a call to this number in a way that is interoperable between operators.

This use case and its extensions have implicitly driven the design of telephony protocols, including that of SIP, which remains in that sense a telephony protocol, as explained in [5].

At first glimpse, OTTs might be seen as replicating this use case. Looking closer, this is not really the case. As a first step, they offer a deep integration of all communication means. For example, Skype provides a full communication suite including address book, presence, IM, video and voice calls. Alice can first look for Bob in her address book and view his presence before calling him. Google has integrated voice into the Gmail webmail service, and with Google Voice proposes a unique personal number, uncorrelated with a device's phone number. The numbering here is partially replaced by the notion of contact; a user does not have to dial phone numbers because they are stored as contacts. Telephony was the fundamental telecommunications service of the telco world; voice is only a part of a communication suite in the OTT environment.

The second step might be more radical. Already, a web browser has become the most widely-used endpoint with which to access digital services. In terms of communication services, web browsers have also been used as voice and video terminals via some proprietary implementations, relying on, for instance, Adobe Flash or plug-ins. The RTC-web effort (Real-Time Communication in WEB-browsers) [6] is continuing to work to standardize the support of real-time interactive communications within the browser, through joint IETF and W3C activities. The IETF work will focus on protocols, while the $\mathrm{W} 3 \mathrm{C}$ will define a set of Javascript APIs to pilot them to set up and control communications.

This last development will probably deeply challenge telephony as we know it. Instead of calling Bob, Alice may browse Bob's Facebook page with her smartphone, and just click to reach him. Bob is notified and takes the communication in his Facebook app. All the control flows remain internal to Facebook; no telephony terminal is needed. The notion of public numbering has disappeared, at least for the people inside our network and using the same social networking platform. As most of our communications are with people from our social network, this new use case could easily become very popular. Communication suites could become an expected component of a social networking application, and, though traditional telephony will remain, its importance will gradually decrease. However, this may force OTTs to leave the status of software editor for the status of telecom service provider, with all the associated duties, such as legal interceptions, universal service or emergency calling.

To face this threat on the telephony market, telcos might choose to block this kind of new telephony application at the network level, for example though IP address filtering, or even DPI (Deep Packet Inspection) mechanisms - eventually offering unblocking as a charged option.

Telcos could also, perhaps simultaneously, evaluate toward a greater separation between the control and network layer, for instance by partnering with some OTT providers. They could even compete with OTT providers by offering unified communications, like they have tried with RCS (Rich Communication Suite). The enterprise market in this situation is more advanced, and major operators are now positioned more as global communication managers than merely telephony operators. By integrating various software and network products, an enterprise can almost never choose a unique product for all its communications needs. BT has, for instance, recently announced the integration of telepresence and visioconference rooms from Cisco, Polycom and Tandberg in a single meeting.

\section{CONCLUSION}

To conclude, we believe that the telco vs OTT battlefield is being conducted both on the control layer and on the devices themselves. Telcos have some key assets.

- Their territorial basis (due to the network physical reality), both for installing equipment and for distributing devices to customers via their points of sales, i.e. their exemplary distribution network in both the technical and marketing senses.

- Their culture of interoperability, both internal (to integrate and operate equipment and devices from various vendors) and external (peering agreements, roaming).

- Their extensive experience with regulation and privacy.

- Their ability to provide unified communications that are not tied to a single range of devices and services.

To their credit, OTTs have also key well-known advantages, such as:

- $\quad$ Their capability to address global markets. 
- Their capability to build service ecosystems.

- Their agility in IT development, partially due to the absence of legacy.

In the future, we will probably witness a layered segmentation in the value networks between OTT and operators, wherein operators provide the network layer and OTT the application layer, the control layer being split according to countries' particularities. Some operators could also federate in order to address global markets, as initiated with the WAC (Wholesale Applications Community) global alliance in the field of application marketplaces. Such initiative could be desirable in other fields, for instance in HD voice, visio-conference or telepresence, to strengthen the telco asset of interoperability.

\section{SOME REFERENCES}

[1] B. Chatras, L. Chong-Chauvot, "IN shortcomings: Myths and realities", in ICIN2001, Bordeaux, France, October 2001

[2] A. Manzalini, R. Minerva, C. Moiso, "The Inner Circle: How to Exploit Autonomic Overlays of Virtual Resources for Creating Service Ecosystems", in ICIN2010, Berlin, Germany, October 2010

[3] E. M. Noam, "The Public Telecommunications Network: A Concept in Transition", Journal of Communication, Vol. 37 issue 1, WilleyBlackwell, March 1987

[4] "Améliorer les offres faites aux consommateurs de services de communications électroniques et postales - Propositions et recommandations", ARCEP, February $2011(\S 12)$

[5] "SIP APIs for Communications on the Web", draft-sinnreich-sip-webapis-01, Internet-Draft, IETF 2010, work in progress

[6] http://rtc-web.alvestrand.com/ 